\title{
BOX-BEHNKEN SUPPORTED DEVELOPMENT AND VALIDATION OF ROBUST RP-HPLC METHOD: AN APPLICATION IN ESTIMATION OF PRAVASTATIN IN BULK AND PHARMACEUTICAL DOSAGE FORM
}

\author{
AJAZ AHMAD ${ }^{\prime}$, MOHAMMAD RAISH ${ }^{2}$ KHALID M. ALKHARFY', \\ KAZI MOHSIN ${ }^{2}$ AND FAIYAZ SHAKEEL *2,3 \\ ${ }^{I}$ Department of Clinical Pharmacy, College of Pharmacy, King Saud University, P.O. Box 2457, Riyadh 11451, Saudi Arabia \\ ${ }^{2}$ Department of Pharmaceutics, College of Pharmacy, King Saud University, P.O. Box 2457, Riyadh 11451, Saudi Arabia \\ ${ }^{3}$ Center of Excellence in Biotechnology Research (CEBR), King Saud University, P.O. Box 2460, Riyadh 11451, Saudi Arabia
}

\begin{abstract}
Pravastatin, a natural bioactive compound inhibits cholesterol biosynthesis which is isolated from fungus by fermentation process. A rapid, sensitive and robust novel reversed phase-HPLC method with UV detection set at $238 \mathrm{~nm}$ was developed and validated for quantification of pravastatin in pure drug form and pharmaceutical formulation (Pravachol). A liquid-liquid extraction technique was applied for the preparation samples and chromatographic separation was performed on Symmetry ${ }^{\circledR} \mathrm{C}_{18}(5 \mu \mathrm{m}, 3.9 \times 150 \mathrm{~mm})$ column with a retention time of $1.95 \mathrm{~min}$ using isocratic elution of mobile phase. The mobile phase used in this work consisted of a mixture of methanol and phosphate buffer $(70: 30 \%, \mathrm{v} / \mathrm{v}, \mathrm{pH} 4)$ which was delivered at a flow rate at $1.5 \mathrm{ml} / \mathrm{min}$. Developed HPLC-UV method was validated for linearity $\left(\boldsymbol{R}^{2}=\mathbf{0 . 9 9 9}\right)$, accuracy, precision, sensitivity, system suitability, selectivity, and robustness. Box-Behnken experimental design was applied in the validation of robustness. Three independent variables such as the composition of mobile phase, flow rate and $\mathrm{pH}$ of mobile phase were investigated. The results indicated that a slight change in the mobile phase composition and flow rate affects the responses, while $\mathrm{pH}$ had no significant effect on the response. Overall, the proposed HPLC-UV method was found to be simple, sensitive, and highly robust for routine analysis of pravastatin.
\end{abstract}

Keywords: Pravastatin; RP-HPLC; Box-Behnken; Robustness; Peak area.

\section{INTRODUCTION}

Statins are well known in reducing the risk of various cardiovascular disorders such as coronary heart disease and strokes. They lower cholesterol levels by inhibiting the enzyme 3-hydroxy-3-methylglutaryl-coanzyme A (HMG-CoA) reductase, at a stage early in the mevalonate pathway such as coenzyme Q10, heme-A, and isoprenylated proteins. ${ }^{1}$ These stages have essential roles in cell biology and humans. ${ }^{2-4}$ The progress of this group of drugs has been one of the major advances in healthcare of humans over the last few decades. Statins have their origins in the discovery of a fungal natural product (compactin or ML-236B), which was shown to have good cholesterol lowering properties. Since compactin itself was not stable enough for clinical use, derivatives with a similar mode of action were prepared to provide useful drugs. ${ }^{5}$

Pravastatin (molecular structure presented in Figure 1) was first isolated as a metabolite product of MLB-236 from canine urine. Afterwards, it was established as a new therapeutic agent in the management and treatment of hypercholesterolemia. ${ }^{6}$ It is obtained by stereoselective hydroxylation of compactin at C-6 position. Industrially, it can be obtained by a two-step production process. In first step, the fermentation of $P$. citrinum is carried out to produces compactin. In second step, the statins are purified by suitable process followed by the lactone ring opening by an addition of sodium hydroxide. After neutralization, the open lactone is converted to pravastatin via biotransformation step with the help of the bacterium Actinomedura sp. ${ }^{7}$ Pravastatin is known to show lowest potential for drug interactions as compared to other statins because it is not metabolized extensively by human cytochromes. ${ }^{6}$

Response surface methodology (RSM) is a statistically designed experimental tool where large numbers of factors are simultaneously studied. ${ }^{8}$, 9 The multivariate methodology has advantages included reduction in the number of experimental runs, improves statistical explanation possibilities and indicates whether parameters interact or not. ${ }^{10}$ Box-Behnken statistical design is known as a multivariate experimental design which is used to optimize the chromatographic parameters and their interaction effects and quadratic effects of the mobile phase composition, flow rate and $\mathrm{pH}$ on the peak area. ${ }^{11}$

The aim of the present work was to develop an RP-HPLC based analysis for pravastatin and assess the robustness of the chromatographic method for the quantitation of pravastatin in bulk and marketed formulation, using response Box-Behnken surface methodology, and determine the analytical parameters that present higher influence in the final results of the analysis using atorvastatin as an internal standard (IS).

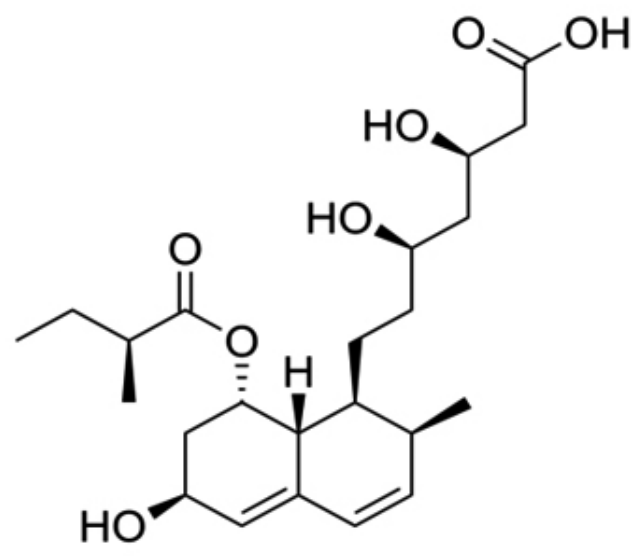

Figure 1: Chemical structure of pravastatin.

\section{EXPERIMENTAL}

\section{Materials}

Pravastatin (purity $\geq 98 \%$ ) and atorvastatin (IS) were purchased from Sigma, USA. HPLC-grade methanol and acetonitrile were obtained from Panreac Chemicals (Barcelona, Spain), potassium dihydrogen phosphate was procured from Winlab Ltd. (Maidenhead, Berkshire, UK) and water was produced in the laboratory by a Milli-Q purification system (Millipore Corp., Billerica, MA, USA). All other reagents used were at least of analytical grade. Milli-QR Gradient A10R filter $(0.22 \mu \mathrm{m}$, Millipore, Moscheim Cedex, France) water was used to prepare the aqueous solutions.

\section{Analytical procedure}

The HPLC system was made up of Shimadzu model SIL 20A auto sampler, model LC 20AD dual piston solvent delivery pump, model SPD 20A dual UV absorbance detector and an online vacuum degasser (Shimadzu, Kyoto, Japan). Two pre injection (blank methanol) flush cycles were run prior to each sample injection, followed by one post-injection flush cycle. The chromatographic identification was carried out at room temperature $\left(25 \pm 1^{\circ} \mathrm{C}\right)$. The mobile 
phase consisted of methanol, acetonitrile and potassium dihydrogen phosphate buffer (20 mM, pH 4.5), in the ratio of 45:30:25 v/v/v, which was delivered isocratically at a flow rate of $1.5 \mathrm{ml} / \mathrm{min}$. Symmetry ${ }^{\mathbb{B}} \mathrm{C} 18(5 \mu \mathrm{m}, 3.9 \times 150$ $\mathrm{mm}$ ) column was utilized to elute the compounds of interest at a $\lambda_{\max }=238$ $\mathrm{nm}$. Signal output was captured using software Shimadzu Lab Solutions32 software, version 3.05 (Tokyo, Kyoto, Japan).

\section{Preparation of standard and quality control samples}

Pravastatin and atorvastatin (IS) standard stock solution $(1 \mathrm{mg} / \mathrm{ml})$ was prepared by dissolving appropriate weighed amount of reference standard in methanol and was kept refrigerated at $4^{\circ} \mathrm{C}$. A series of working standard solutions were prepared by subsequent dilution of the stock standard solutions in methanol to reach concentration ranges of $0.1-50 \mu \mathrm{g} / \mathrm{ml}$ for pravastatin. Calibration curves were constructed by plotting the area of the peak against the known concentration. The known concentrations used to make the calibration curves were $0.1,0.5,1,5,10,15,20,25$ and $50 \mu \mathrm{g} / \mathrm{ml}$ for pravastatin. Each concentration point was added with atorvastatin $(10 \mu \mathrm{g} / \mathrm{ml})$. QC samples were prepared at four different levels: $0.1 \mu \mathrm{g} / \mathrm{ml}$ lower limits of quantification (LLOQ), $0.3 \mu \mathrm{g} / \mathrm{ml}$ low quality control (LQC), $20 \mu \mathrm{g} / \mathrm{ml}$ middle quality control (MQC), and $40 \mu \mathrm{g} / \mathrm{ml}$ high quality control (HQC). Sample preparation and analysis were conducted at room temperature $\left(25 \pm 1^{\circ} \mathrm{C}\right)$. Linear regression tests were performed via the least squares method using Microsoft Excel 2007. The equation for the respective calibration curve was extrapolated to allow for determination of all subsequent calculations.

\section{Method validation}

After the chromatographic conditions were optimized, the method was validated according to International Conference on Harmonization (ICH) guidelines ${ }^{12}$, in order to evaluate the method for selectivity, linearity of response, accuracy, precision, recovery, limit of detection and quantification, robustness and stability of analytes during processing and storage.

The capability of the assay to give the analyst data that is directly proportional to the amount of analyte that the sample contains. The column was equilibrated with the mobile phase for at least $1 \mathrm{~h}$ before sample injection. The linearity was evaluated by the construction of calibration curves using standard solutions with concentrations ranging from $(0.1-50 \mu \mathrm{g} / \mathrm{ml})$. Quality control samples at four different concentration levels $0.1 \mu \mathrm{g} / \mathrm{ml}$ lower limit of quantitation (LLOQ), $0.3 \mu \mathrm{g} / \mathrm{ml}$ low quality control (LQC), $20 \mu \mathrm{g} / \mathrm{ml}$ middle quality control (MQC) and $40 \mu \mathrm{g} / \mathrm{mL}$ high quality control (HQC) were used. Six replicates at each QC concentration level were analyzed along with an independently prepared calibration curve and the assays were performed on three different days.

The accuracy and the precision for each QC level were evaluated by calculating the recovery percent and relative standard deviation (RSD) of the measured concentrations, respectively. The accuracy was to be within $\pm 10 \%$ and the precision, (intra- and inter-day) was required to be less than $15 \%$.

The percent recovery study was conducted by the spiking method in the sample preparation. Pre-analyzed samples were spiked with extra 50,100, and $150 \%$ of pravastatin standard and the mixtures were re-analyzed by the proposed method. The experiment was conducted in triplicates.

The lowest amount of the analyte that the proposed method is able to detect is known as the limit of detection (LOD). Similarly, the limit of quantification (LOQ) is the minimum amount of the analyte that is being measured that the suggested procedure is able to detect with acceptable degrees of accuracy and precision. Determination of LOD and LOQ, blank methanol (without pravastatin) was injected in triplicate for peak area calculations. LOD and LOQ were determined from the slope (S) of the calibration curve and SD of the response for the blank samples using the following formulae:

$$
\begin{aligned}
& \text { LOD }=3.3 \times \frac{S D}{S} \\
& L O Q=10 \times \frac{S D}{S}
\end{aligned}
$$

The selectivity of the method was checked by injecting solutions of pravastatin and blank methanol. It was observed that the sharp peaks of pravastatin were obtained at retention time $1.95 \mathrm{~min}$; these peaks were not obtained from blank methanol solution. The specificity of the method was assessed by comparing chromatograms obtained from drug standards with that obtained from the formulation. The retention times of the pravastatin standard and the extracted pravastatin from the marketed formulation were found to be same, indicating the method was specific and selective because there was no interference from the pharmaceutical dosage form.

\section{Robustness}

Robustness of the current method was performed by introducing very small changes in the analytical methodology at a single concentration level (1 $\mu \mathrm{g} / \mathrm{ml}$ ). Robustness of the proposed method was determined by changing in three parameters (i.e., mobile phase, flow rate, and $\mathrm{pH}$ of the buffer solution). Box-Behnken response surface design was used in the current investigation to evaluate the robustness of the proposed method. The design concurrently assessed the effects of the three tested parameters on retention time of pravastatin. Design-Expert 8.0 software (Stat Ease Stat-Ease, Inc., Minneapolis, MN, USA) was used to evaluate the result. Three-dimensional graphs represented the retention time dependence on mobile phase concentration, flow rate of the mobile phase and $\mathrm{pH}$ of the buffer solution. Effects of the selected factors were evaluated over a range of conditions by determining the maximum retention time response of the pravastatin.

\section{Analysis of pravastatin in a marketed formulation}

The current developed chromatographic method was used for quantification of pravastatin in a pharmaceutical formulation. The marketed formulation of pravastatin $40 \mathrm{mg}$ was procured from BMS (London, UK) and evaluated for the amount of pravastatin present in the formulation. Twenty tablets were weighed and crushed to a fine powder. An amount equivalent to $50 \mathrm{mg}$ of pravastatin was accurately weighed and transferred to a $50 \mathrm{ml}$ volumetric flask, dissolved in $50 \mathrm{ml}$ methanol, sonicated for $10 \mathrm{~min}$ for complete extraction of the pravastatin. The solution was centrifuged at $2500 \times \mathrm{g}$ for $10 \mathrm{~min}$, and the clear supernatant was collected and filtered through a $0.45 \mu \mathrm{m}$ membrane filter. The solution was diluted further to get concentrations within the linear range of the calibration curve $(0.1-50 \mu \mathrm{g} / \mathrm{ml})$. Aliquots $(20 \mu \mathrm{l})$ of the samples were injected into the HPLC system for analysis.

\section{RESULTS AND DISCUSSION}

\section{Method development and linearity}

The present chromatographic method was developed and validated according to ICH guidelines and statistical analysis was performed using Microsoft Excel 2007 (Microsoft Corp, Seattle, WA, USA). The linear regression analysis indicated a good linearity over a wide concentration range $(0.1-50 \mu \mathrm{g} / \mathrm{ml})$ with regard to peak area ratio. To optimize the HPLC conditions for an efficient separation of pravastatin, various factors such as mobile phase composition, flow rate, column temperature and $\mathrm{pH}$ of the mobile phase were investigated. It was observed that $\mathrm{C} 18$ column $(3.9 \times 150 \mathrm{~mm})$ with particle size 5 um gave the most suitable resolution. The suitability of mobile phase combination, flow rate, and $\mathrm{pH}$ was decided on the basis of linearity, sensitivity, system suitability, selectivity, lesser time required for analysis (low retention time), peak parameters, and ease of preparation. The mobile phase compositions were prepared with appropriate ratios of acetonitrile, methanol, water and phosphate buffer. It was observed that the best resolution of pravastatin was attained with a mobile phase composed of methanol and phosphate buffer at $70: 30, \mathrm{v} / \mathrm{v}$ ratio at $\mathrm{pH} 4$ and a flow rate of $1.5 \mathrm{ml} / \mathrm{min}$. The highest sensitivity with minimum interference for both the drug and IS was accomplished at $238 \mathrm{~nm}$.

\section{Method validation}

The RP-HPLC method was validated for selectivity, linearity, accuracy, precision, robustness, limits of detection (LOD) and quantification (LOQ). No significant interference from blank methanol was observed at the corresponding retention times of pravastatin as shown in Figure $2 \mathrm{~A} \& \mathrm{~B}$, demonstrating the selectivity of the method.

A calibration curve of pravastatin concentration in methanol was linear over the range of $0.1-50 \mu \mathrm{g} / \mathrm{ml}$ for three consecutive days with mean coefficient of determination $\left(\boldsymbol{R}^{2}\right)$ of $>0.999$ (Table I). The linearity range was evaluated between the LLOQ and the HQC which representing the lowest and the highest concentration points of the established calibration curves. Calibration was performed with 10 standard points at 0.1 (LLOQ), 0.3 (LQC), 0.5, 1, 5, 15, 
20 (MQC), 25, 40 (HQC) and $50 \mu \mathrm{g} / \mathrm{ml}$ of pravastatin. Calibration curve was generated using the analyte to internal standard peak area ratio (Table I). The LOD and LOQ values of pravastatin were obtained as 0.022 and $0.068 \mathrm{ng} / \mathrm{ml}$, respectively.
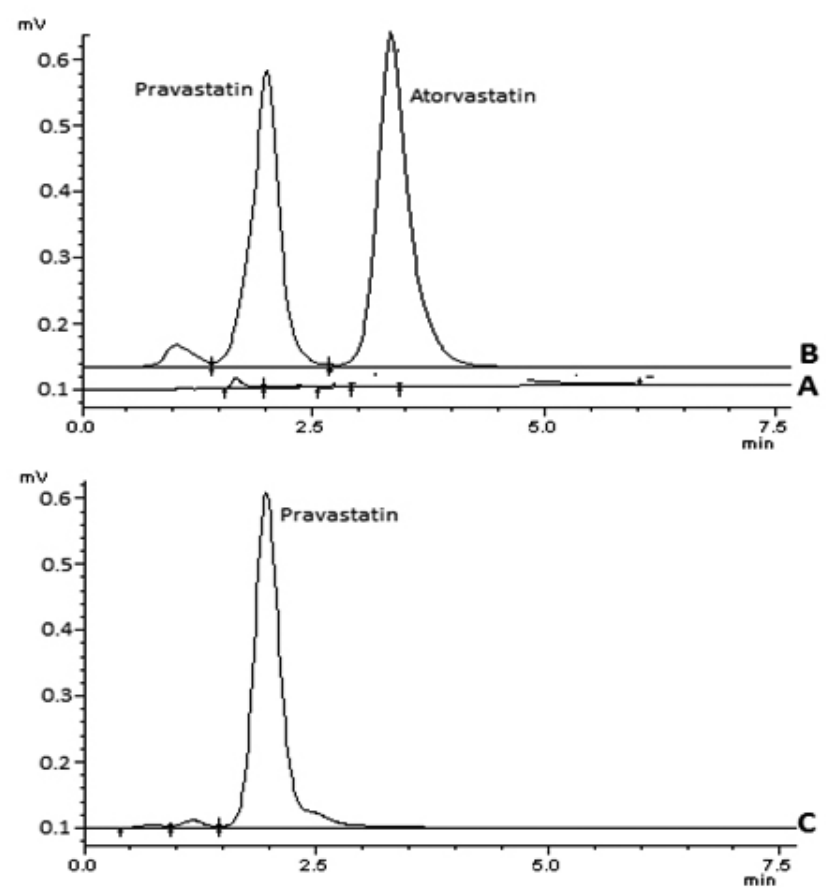

Figure 2: A typical chromatograms of (A) blank methanol (B) Pravastatin standard solution $(10 \mu \mathrm{g} / \mathrm{ml}, \mathrm{RT}=1.95)$ and atorvastatin (IS) $(15 \mu \mathrm{g} / \mathrm{ml}, \mathrm{RT}=$ $3.74)$ and $(\mathrm{C})$ pravastatin $(5 \mu \mathrm{g} / \mathrm{ml})$ marketed formulation.

Table 1: Precision and accuracy of pravastatin calibration curve points in standard drug.

\begin{tabular}{|c|c|c|c|}
\hline Pravastatin & & & \\
\hline Added Conc. $(\mu \mathrm{g} / \mathrm{ml})$ & Mean $\pm \mathrm{SD}$ & $\begin{array}{c}\text { Precision }(\mathrm{CV} \\
\%)\end{array}$ & Accuracy $(\%)$ \\
\hline 0.1 & $0.10 \pm 0.00$ & 2.03 & 95.50 \\
\hline 0.50 & $0.54 \pm 0.04$ & 8.32 & 98.00 \\
\hline 1 & $0.98 \pm 0.06$ & 5.55 & 98.00 \\
\hline 5 & $5.11 \pm 0.16$ & 3.11 & 100.00 \\
\hline 10 & $11.05 \pm 0.28$ & 2.54 & 91.45 \\
\hline 15 & $14.37 \pm 0.60$ & 4.06 & 101.37 \\
\hline 20 & $19.48 \pm 0.59$ & 2.95 & 100.53 \\
\hline 25 & $25.34 \pm 0.35$ & 1.41 & 99.64 \\
\hline 50 & $51.22 \pm 0.64$ & 1.23 & 96.66 \\
\hline
\end{tabular}

Intra-assay precision and accuracy of proposed analytical method were investigated by analysis of six replicates at four different QC concentrations and results are presented in Table 2 . The precision of propose analytical method was evaluated in terms of percent of the relative standard deviation (\% RSD) which was observed as 2.68 to $5.26 \%$. While, the intra-assay accuracy values for pravastatin were obtained as 97.80 to $101.26 \%$. The inter-assay precision and accuracy were evaluated by analysis of six replicates of each of the four QC concentrations in each of three assay runs $(n=18$; Table 2$)$. Reproducibility of the method exhibited inter-assay precision ranging from 1.37 to $3.06 \%$ and Inter-assay accuracy values ranged from 97 to $102.68 \%$. The results were within the acceptable limits for precision and accuracy. The method was used for extraction and subsequent analysis of pravastatin after spiking with 50,100 , and $150 \%$ of additional drug. Extraction recovery of pravastatin was determined by comparing the peak areas from the extracted samples with unextracted standards that represent $100 \%$ recovery. The recovery of pravastatin was found to be 98.26 to $103.62 \%$ (Table 3).

Chromatograms from six different batches of blank methanol were compared to those form the corresponding samples to evaluate selectivity. No significant interference from the blank methanol was observed at the corresponding retention times of pravastatin as shown in Figure $2 \mathrm{~A} \& \mathrm{~B}$, demonstrating the selectivity of the method.

Table 2: Intra and inter-day precision and accuracy of Pravastatin in standard drug.

\begin{tabular}{|c|c|c|c|}
\hline Pravastatin & & & \\
\hline Intra-day $(n=6)$ & & & \\
\hline Added Conc. $(\mu \mathrm{g} / \mathrm{ml})$ & Mean \pm SD & Precision $(\mathrm{CV} \%)$ & Accuracy $(\%)$ \\
\hline 0.1 & $0.10 \pm 0.01$ & 5.26 & 97.80 \\
\hline 1.5 & $1.49 \pm 0.21$ & 2.91 & 99.56 \\
\hline 20 & $21.03 \pm 0.58$ & 2.68 & 98.32 \\
\hline 40 & $39.86 \pm 1.95$ & 3.59 & 101.26 \\
\hline Inter-day $(\mathrm{n}=18)$ & & & \\
\hline 0.1 & $0.10 \pm 0.00$ & 1.37 & 97.00 \\
\hline 1.5 & $1.51 \pm 0.12$ & 2.91 & 102.68 \\
\hline 20 & $19.91 \pm 0.35$ & 3.06 & 98.39 \\
\hline 40 & $41.36 \pm 1.28$ & 2.39 & 102.59 \\
\hline
\end{tabular}

Table 3: Results from study of analytical recovery $(n=6)$ of Pravastatin.

\begin{tabular}{|c|c|c|}
\hline $\begin{array}{c}\text { Pravastatin } \\
\text { Excess drug added to the analyte } \\
(\%)\end{array}$ & $\begin{array}{c}\text { Theoretical content } \\
(\mu \mathrm{g})\end{array}$ & Recovery $(\%)$ \\
\hline 0 & 1 & $98.26 \pm 0.74$ \\
\hline 50 & 1.5 & $100.25 \pm 0.61$ \\
\hline 100 & 2 & $101.89 \pm 0.38$ \\
\hline 150 & 3.5 & $103.62 \pm 0.12$ \\
\hline
\end{tabular}

\section{Robustness}

The Box-Behnken experimental design was applied to investigate the robustness of the developed method and results are presented in Figure 3. RSM is an effective statistical technique which is applied to investigate and optimize complex processes. This design offers several advantages over conventional methods of optimization. ${ }^{11,13,14}$ This design also produces a large quantity of information and being considered as more economical approach because; less number of experiments are required for observing the interaction of the independent variables on the measured responses (dependent variables). ${ }^{13}$ Center points, two or more repetitions, are generally carried to measure pure error. This permits the calculation of the response of intermediate levels which ultimately enables an evaluation of the system performance at any experimental point. ${ }^{11}$
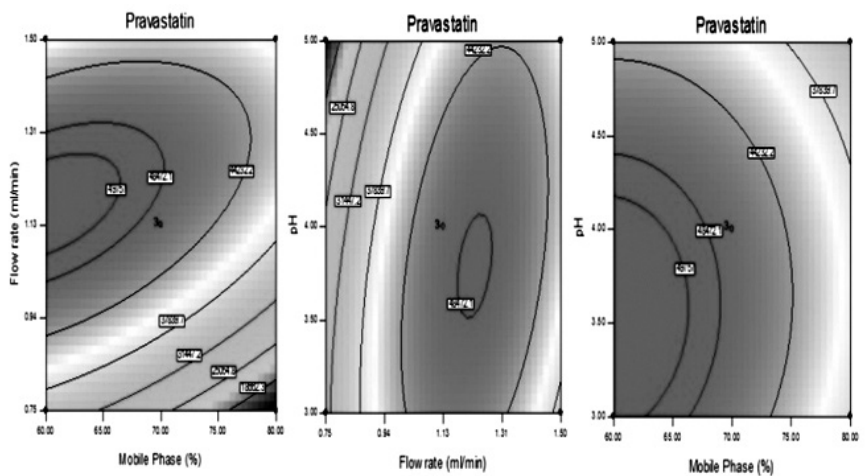

Figure 3: Response-surface contour graphs showing the effect of methanol versus flow rate, $\mathrm{pH}$ versus flow rate and mobile phase versus $\mathrm{pH}$. 
Table 4: Recovery of pravastatin in marked formulation tablet by developed RP-HPLC Method.

\begin{tabular}{|c|c|c|}
\hline \multirow{2}{*}{ Label claim } & Amount found (mg) & Recovery (\%) \\
\hline \multirow{3}{*}{$40 \mathrm{mg}$} & 39.65 & 98.65 \\
\cline { 2 - 3 } & 41.31 & 100.28 \\
\cline { 2 - 3 } & 40.02 & 101.37 \\
\cline { 2 - 3 } & 40.64 & 100.24 \\
\cline { 2 - 3 } & 42.06 & 102.08 \\
\hline Mean (\%) & 39.48 & 98.72 \\
\hline CV (\%) & $40.52 \pm 0.89$ & $100.22 \pm 0.62$ \\
\hline
\end{tabular}

Table 5: The CCRD Response surface design and corresponding response values.

\begin{tabular}{|c|c|c|c|c|c|c|}
\hline & & & & Peak & & \\
\hline Run & MP & Flow rate & $\mathrm{pH}$ & Actual & Predicted & Residual \\
\hline 1 & 60 & 0.75 & 4 & 34901.3 & 34633.48 & 267.825 \\
\hline 2 & 80 & 0.75 & 4 & 10806.8 & 12269.78 & -1462.97 \\
\hline 3 & 60 & 1.5 & 4 & 41319.9 & 39856.93 & 1462.97 \\
\hline 4 & 80 & 1.5 & 4 & 39151.9 & 39419.73 & -267.82 \\
\hline 5 & 60 & 1.125 & 3 & 49863.8 & 50252.30 & -388.5 \\
\hline 6 & 80 & 1.125 & 3 & 39055.8 & 37713.50 & 1342.3 \\
\hline 7 & 60 & 1.125 & 5 & 41938.4 & 43280.70 & -1342.3 \\
\hline 8 & 80 & 1.125 & 5 & 33407.1 & 33018.60 & 388.5 \\
\hline 9 & 70 & 0.75 & 3 & 30430.4 & 30309.73 & 120.675 \\
\hline 10 & 70 & 1.5 & 3 & 35187.6 & 36262.08 & -1074.47 \\
\hline 11 & 70 & 0.75 & 5 & 15316.6 & 14242.13 & 1074.47 \\
\hline 12 & 70 & 1.5 & 5 & 40542.5 & 40663.18 & -120.675 \\
\hline 13 & 70 & 1.125 & 4 & 47709.7 & 47349.67 & 360.03 \\
\hline 14 & 70 & 1.125 & 4 & 47889.8 & 47349.67 & 540.13 \\
\hline 15 & 70 & 1.125 & 4 & 46449.5 & 47349.67 & -900.16 \\
\hline
\end{tabular}

In the current study, three factors were evaluated: percentage $v / v$ of methanol (A); flow rate (B) and $\mathrm{pH}$ of mobile phase (C) and results are presented in Table 5. RSM methodology was applied to optimize these independent variables. In the current study, this experimental design was based on a 33 factorial design, three central points, leading to 15 sets of experiments, enabling each measured response to be optimized. The outcomes were examined with the help of a Box-Behnken experimental design using the Design Expert software (V 8.0.7.1). Standard order was followed for all experiments runs to diminish the effects of uncontrolled factors that may introduce a partiality in the predicted response. The levels of the three independent variables evaluated in this design are presented in Table 5 . The quality of the fitted model was expressed in terms of the coefficient of determination $\left(\boldsymbol{R}^{2}\right)$, and analysis of variance (F-test) was checked for statistical significance. The best processing conditions were attained by using graphical and numerical analysis based on the criteria of the desirability function and the response surface. The experiment was finally repeated under the optimum values as per the point prediction tool of RSM methodology for percentage $(v / v)$ of methanol (A), flow rate $\mathrm{ml} / \mathrm{min}(\mathrm{B})$ and $\mathrm{pH}$ of the mobile phase $(\mathrm{C})$, which should result in sharp peak, maximum peak area and no change in retention time. The polynomial second-order equation expresses experimental relationship of model and the input variables with interaction terms was fitted between achieved results. The fitted model equation is:
Analysis of variance (ANOVA) was used to assess the design data and the importance of the regression coefficients that were estimated by consistent $\mathrm{p}$-values and is presented in Table 6. The assessment of experimental error variance permits the significance of the coefficients to be evaluated. The ANOVA pointed out that the current model proposed were significant, thus describing that the change in the design response was due to change of factor levels. P-values of each model terms determined that three linear coefficients (A, B and C), three quadratic coefficients $\left(\mathrm{A}^{2}, \mathrm{~B}^{2}\right.$ and $\left.\mathrm{C}^{2}\right)$ and two interactive coefficients $(\mathrm{AC}$ and $\mathrm{BC})$ were significant and indicate the pattern of the interactions between the tested variables. The model F-value of 82.21 indicated that the model was significant at $p<0.0001$. The reliability of the fit of the model was assessed by the estimation co-efficient $\left(\boldsymbol{R}^{2}=\mathbf{0 . 9 9 3}\right)$, and adjusted determination co-efficient $\left(\boldsymbol{R}^{2} \mathbf{a}=\mathbf{0 . 9 8 1}\right)$ and co-efficient of variance $(\mathrm{CV}=$ $4.18 \%$ ). The low values of $\mathrm{CV}$ indicate that the abnormalities between tested and predicted values are low and it not only exhibited a high degree of precision but also had a good reliability in experiments.$^{15}$ Adequate precision greater than 4 is desirable and the ratio was found to be 30.15 , which indicates an adequate signal and confirm that this model is significant for this extraction process. ${ }^{16}$

Contour plots for the tested responses were made based on the polynomial model to evaluate the change of the response surface. Additionally, these are helpful to appreciate the relationship among the dependent and independent factors. Subsequently the model has more than two factors; one factor was kept constant for each plot. As shown in Figure 3(A-C), the design produces contour graphs by plotting the response model against two of the factors, while the third is held constant at a specified level, usually the proposed optimum. Figure 3A shows a graphical representation of the response surface for variation of percentage of methanol, and flow rate, while the $\mathrm{pH}$ is maintained constant at its optimum of 4.0. An increase in the flow rate results in a decrease of the observed peak area ratio $(\mathrm{Y})$, while the percentage of organic modifier had no important effect on the peak area. Analogous interpretation may be derived by examining Figure $3 \mathrm{C}$ that plots the factors flow rate versus $\mathrm{pH}$. In Figure 3B, where the factor flow rate is maintained constant, the method can be considered robust for the studied experimental response. The optimum conditions were established to be percentage of methanol (65), flow rate (1.20 $\mathrm{ml} / \mathrm{min}$ ) at $\mathrm{pH} 4$ gives the maximum peak area (49896) for the pravastatin. On the above-mentioned optimum conditions, the experiment was conducted in triplicates for the validation. Experimental conditions were selected according to the response diagrams by using point prediction tool of the software. These optimized values of tested parameters were validated under similar conditions $(n=6)$, an average peak area (48935) of pravastatin was obtained in an optimized robust conditions with $98.07 \%$ validity. The results of analysis confirmed that the response model was adequate for reflecting the expected optimization, and the model was satisfactory and accurate.

Estimation of pravastatin in pharmaceutical dosage form

The proposed analytical method was applied to the quantitative analysis of pravastatin in marketed formulation (Pravchol ${ }^{\circledR}$ tablets). A representative chromatogram is presented in Figure 2C. The absence of additional peaks in the chromatogram indicated no interference of the formulation excipients used in the tablet. The results obtained are presented in Table 4, showed high percentage recovery with low CV (\%) values of the six replicates, which confirm the method is suitable for routine analysis of pravastatin in pharmaceutical preparations, with a mean percentage recovery from tablet of $100.22 \%$ for pravastatin. The results were in good agreement with the label claim of the product.

\section{CONCLUSION}

A simple, selective, rapid, highly sensitive and rapid HPLC method was developed and validated successfully for the estimation of pravastatin in in bulk as well as in pharmaceutical formulations. The method using methanol and phosphate buffer mixture as mobile phase exhibited a decent chromatographic separation. The method was shown to be precise, accurate, and linear over the concentration range tested $(0.1-50 \mu \mathrm{g} / \mathrm{ml})$ with a correlation coefficient $\left(\boldsymbol{R}^{2}\right)$ of 0.999. Application of factorial design using Box-Behnken for validation of robustness testing indicated that a change in flow rate and mobile phase ratio has an effect on peak area. Hence, special attention is required for strict monitoring of the aforementioned two factors during chromatographic testing. Consequently, the developed method is evidenced to be rapid, simple, selective and robust for routine analysis of pravastatin in bulk as well as in pharmaceutical formulations. 
Table 6: Analysis of variance and regression coefficients of calculated surface quadratic model for peak area of pravastatin.

\begin{tabular}{|c|c|c|c|c|c|c|c|}
\hline Source & $\begin{array}{l}\text { Coefficient } \\
\text { estimate }\end{array}$ & Sum of squares & Mean Square & Standard error & Degree of freedom & F Value & $P$ Value \\
\hline Model & 47349.67 & 1760553098 & 195617010.9 & 890.66 & 9 & 82.20 & $<0.0001$ \\
\hline A & -5700.22 & 259940520.4 & 259940520.4 & 545.42 & 1 & 109.23 & 0.0001 \\
\hline B & 8093.35 & 524018513.8 & 524018513.8 & 545.42 & 1 & 220.19 & $<0.0001$ \\
\hline $\mathrm{C}$ & -2916.62 & 68053611.13 & 68053611.13 & 545.42 & 1 & 28.60 & 0.0031 \\
\hline $\mathrm{AB}$ & 5481.62 & 120192850.6 & 120192850.6 & 771.34 & 1 & 50.50 & 0.0009 \\
\hline $\mathrm{AC}$ & 569.17 & 1295840.722 & 1295840.72 & 771.34 & 1 & 0.54 & 0.4937 \\
\hline $\mathrm{BC}$ & 5117.17 & 104741919.9 & 104741919.9 & 771.34 & 1 & 44.01 & 0.0012 \\
\hline $\mathrm{A} 2$ & -2553.84 & 24081705.38 & 24081705.38 & 802.83 & 1 & 10.12 & 0.0245 \\
\hline B2 & -13250.84 & 648313533.4 & 648313533.4 & 802.83 & 1 & 272.42 & $<0.0001$ \\
\hline $\mathrm{C} 2$ & -3729.54 & 51358198.61 & 51358198.61 & 802.83 & 1 & 21.58 & 0.0056 \\
\hline Residual & - & 11899241.25 & 2379848.25 & - & 5 & - & - \\
\hline Lack of Fit & - & 10667573.21 & 3555857.73 & - & 3 & 5.77 & 0.1512 \\
\hline Pure Error & - & 1231668.04 & 615834.02 & - & 2 & & \\
\hline Corr Total & - & 1772452339 & & - & 14 & & \\
\hline $\begin{array}{c}\text { Adeq. } \\
\text { Precision }\end{array}$ & 30.15 & & & & & & \\
\hline
\end{tabular}

A:Mobile Phase Composition, B:Flow rate, C:pH

\section{ACKNOWLEDGEMENT}

The authors would like to extend their sincere appreciation to the Deanship of Scientific Research at King Saud University for its funding the work through the research group no. RG-1435-017.

\section{REFERENCES}

1.- H. Buhaescu, Clin. Biochem. 40, 575 (2007).

2.- J.L. Goldstein, M.S. Brown, Nature. 343, 425 (1999).

3.- F. Mach, Circulation. 109, 1115 (2004).

4.- J. Keyhani, E. Keyhani, FEBS Lett. 93, 271 (1978).

5.- G.M. Cragg, D.J. Newman, Biochim. Biophys. Acta 1830, 3670 (2013).

6.- J. Barrios-González, R.U. Miranda, Appl. Microbiol. Biotechnol. 85, 869 (2010).

7.- A. Ahmad, M. Mujeeb. R. Kapoor, B.P. Panda, Chem. Pap. 67, 667 (2013).

8.- D. Montgomery, Design and analysis of experiment" New York, John Wiley and Sons (1991).
9.- L. Shiow-Ling, C. Wen-Chang, Enz. Microb. Technol. 21, 436 (1997).

10.- R.H. Myers, and D.C. Montgomery DC, Response Surface Methodology: Process and Product Optimization Using Designed Experiments, $2^{\text {nd }}$ ed. Wiley, New York (2002).

11.- S.L. Ferreira, R.E. Bruns, H.S. Ferreira, G.D. Matos, J.M. David, G.C Brandao, E.G. da Silva, L.A. Portugal, P.S. dos Reis, A.S. Souza, W.N. dos Santos, Anal. Chim. Acta 597, 179 (2007).

12.- ICH, ICH Draft Guidelines on Validation of Analytical Procedures: Definition and Terminology, Switzerland, Federal Register, IFPMA (1995).

13.- M.A Bezerra, R.E. Santelli, E.P. Oliveira, L.S. Villar, L.A. Escaleira, Talanta 76, 965 (2007).

14.- R.L. Mason, R.F. Gunst, J.L. Hess, Statistical Design and Analysis of Experiments. Hoboken, NJ: Wiley; p. 586 (2003).

15.- T. Lundstedt, E. Seifert, L. Abramo, B. Thelin, A. Nyström, J. Pettersen, R. Bergman, Chem. Intel. Lab. Sys. 42, 3 (1998).

16.- Q.K. Beg, V. Sahai, R. Gupta, Proc. Biochem. 39, 203 (2003). 